\title{
Movimientos sociales y política en el Perú de hoy
}

Anabi Durand Guevara*

Resumen: El presente artículo analiza la dinámica de movilización y representación políicca emprendida por los principales movimientos sociales del actual Perú, los cuales se desenvuelven en un contexto signado aún por las reformas estructurales, los cambios constitucionales y la persistencia de consensos ideológicos autoritarios, que denominamos "posfuijimorismo". Se aborda así el proceso por el cual estos movimientos generan confluencias de plataformas y encuentros de sentido, tanto entre organizaciones como entre actores político-institucionales que incursionan en la competencia electoral.

Palabras Clave: Movimientos sociales, Representación políitica, Fujimorismo, Presión extractiva, Conflictividad social, Democracia.

ABSTRACT: This article analyzes the dynamic of political mobilization and representation developed by the major social movements in present-day Peru, which unfold in a context still marked by structural reforms, constitutional changes and the persistence of an authoritarian ideological consensus that give shape to the so-called "post-Fujimori" period. Thus, this article addresses the process by which these movements create convergences in platforms and come together as much as between organizations as with institutionalized political actor such as parties, venturing into electoral competition.

Key words: Social Movements, Political Representation, Fujimorism, Extractive Pressure, Social Unrest, Democracy.

Instituto de Estudios Peruanos (anahi@iep.org.pe). 
INTRODUCCIÓN

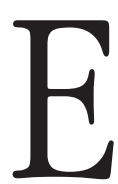

n las últimas décadas, Latinoamérica en general y Perú, en particular, han experimentado sustantivas transformaciones con efectos directos en las demandas de los actores sociales, sus plataformas políticas, discursos y marcos identitarios. Hechos como la profundización de la política económica neoliberal o la persistencia de altas tasas de pobreza y marginalidad, revelan los límites del actual modelo económico y de desarrollo, interpelando a los pueblos y organizaciones respecto a cómo concretar sus demandas, ya sea desde la vía contenciosa o la disputa por puestos de poder. Se articulan así dinámicas complejas, en las que la decisión de los movimientos sociales de combinar protesta y participación electoral no es necesariamente un proceso de confluencia armoniosa, originando a menudo tensiones y debates que expresan cambios importantes en las formas de participar y entender la política.

El presente artículo busca exponer un panorama general de la dinámica de movilización y representación política emprendida por los principales movimientos sociales presentes en Perú, los cuales se desenvuelven en un contexto signado aún por las reformas estructurales, los cambios constitucionales y la persistencia de consensos ideológicos autoritarios, que configuran lo que denominamos "posfujimorismo". Luego de un repaso general del escenario histórico de desenvolvimiento, analizamos los principales discursos, marcos identitarios y reivindicaciones de los actores sociales que surgen o se reconfiguran a fines de los noventa, superada la etapa de conflicto armado, posterior pacificación y en plena crisis del último gobierno de Fujimori. En tercer lugar, abordamos el proceso por el cual estos movimientos generan confluencias de plataformas y encuentros de sentido tanto entre organizaciones cuanto con actores político institucionales como los partidos, incursionando en la competencia electoral. Finalmente, ensayamos un acercamiento al actual momento de movilización, signado por la llegada al poder de Ollanta Humala y su alineamiento con el consenso neoliberal en detrimento de las promesas reformistas que le valieron el apoyo de las organizaciones. Se abre así un nuevo momento en la acción política de los movimientos sociales, distanciados temporalmente 
de los principales partidos políticos pero asumiendo a la vez nuevos desafíos en la apuesta por transformar un orden social que continúa siendo injusto y desigual para las mayorías.

\section{I. ¿VEINTE AÑOS SON NADA? 1992-2012 POR QUÉ HABLAR DE POSFUJIMORISMO}

Han pasado poco más de dos décadas desde que el 5 de abril de 1992 Alberto Fujimori ejecutó el denominado "autogolpe" copando los poderes del Estado y promoviendo la promulgación de una nueva Constitución. A pesar del tiempo transcurrido, podemos afirmar que vivimos todavía en una sociedad "posfujimorista" expresada en tres aspectos profundamente vinculados entre sí. Por un lado, el mantenimiento del andamiaje económico neoliberal profundizado a partir del autogolpe, por otro lado las trabas constitucionales que legitiman y dan estabilidad al modelo, y finalmente la preeminencia de un consenso autoritario conservador respecto a la historia reciente y a las formas de encarar la protesta social.

En cuanto al estableciemiento del modelo neoliberal, el gobierno de Fujimori aseguró concentrar el poder en el Ejecutivo a expensas del Congreso y del Poder Judicial, y al interior del mismo Ejecutivo a través del Ministerio de Economía y Finanzas (MEF) y el aparato de seguridad. Esta manera de aplicar el neoliberalismo facilitó lo que Francisco Durand ${ }^{1}$ denomina "la captura del Estado" por parte del sector privado, las corporaciones nacionales y multinacionales, allanando el camino hacia la extrema concentración del poder económico en torno a una pequeña élite empresarial coludida con la mafia fujimontesinista. Justamente, un eje clave en el proceso de reformas neoliberales emprendidas fueron las privatizaciones de las empresas estatales, aceleradas desde 1994 y seguidas por una ola de adquisiciones, concesiones y nuevas inversiones, que continuaron con fuerza durante las décadas siguientes, aumentando la dependencia del Estado sobre el capital. Esto incluyó empresas de servicios públicos creadas du-

1 Francisco Durand, "El debate sobre la captura del Estado Peruano", en Eduardo Toche [comp.], Perú boy; la gran continuidad, Lima, Centro de Estudios y Promoción del Desarrollo, 2012. 
rante el gobierno de Velasco, como la Compañía Peruana de Teléfonos o la Empresa Nacional de Telecomunicaciones que favorecieron a monopolios privados en procesos que no fueron materia de deliberación pública. ${ }^{2}$ Por otro lado, se privatizaron y concesionaron empresas estatales como MINERO PERÚ o HIERRO PERÚ, pasando a manos del capital extranjero en el marco de la promulgación de la Ley General de Minería publicada en junio de 1992 en un contexto de alta corrupción y discrecionalidad, cuyo resultado fue que algunas multinacionales y grupos económicos locales controlaron las comunicaciones, la banca, los fondos de pensiones, la minería y expandieron su importancia en el sector de hidrocarburos.

Otro aspecto central del modelo que se ha mantenido, e incluso que se ha intensificado, es la denominada reprimarización económica, a partir de la cual el país experimenta un retorno a la economía "primario-exportadora" con el consecuente retroceso del sector manufacturero y la pérdida de importancia del proyecto industrializador. Se prioriza así la exportación de recursos naturales, principalmente minerales, gas y petróleo y se incrementa la presión sobre los territorios donde éstos se ubican. Como señala Anthony Bebbington ${ }^{3}$ ya en el periodo 1990-1997 mientras la inversión en exploración minera en un ámbito mundial creció 90\%, en América Latina aumentó 400\%. Para el caso peruano, según cifras del minem, en los últimos 10 años el valor de las exportaciones mineras se ha multiplicado siete veces y ha pasado de poco más de 3 mil millones de dólares en 1999 a la cifra récord histórica de 21.723 millones durante el 2010.

Para garantizar la aplicación y permanencia de las reformas emprendidas, se necesitaba de un marco normativo institucional que brindara estabilidad al modelo, emitiendo señales de confianza al capital transnacional y otros actores privados que invertían en Perú. La Constitución de 1993, elaborada por un cuestionado Congreso Constituyente Democrático y avalada por un dudoso

2 María Isabel Remy, "Democracia y desigualdad: poderes fácticos, régimen político y ciudadanías diferenciadas", en Julio Cotler y Ricardo Cuenca [eds.], Las desigualdades en el Perú, balances críticos, Lima, Instituto de Estudios Peruanos, 2011.

3 Anthony Bebbington, "Elementos para una ecología política para los movimientos sociales y el desarrollo territorial en zonas mineras", en Anthony Bebbington [ed.], Minería, movimientos sociales y respuestas campesinas. Una ecología política de transformaciones territoriales, Lima, Instituto de Estudios Peruanos, 2007. 
referéndum, cumplió con estos objetivos, al otorgar a las reformas la legitimidad necesaria. Se instauró así un marco legal y normativo abiertamente favorable a la inversión privada que minimiza el rol del Estado y limita las posibilidades de ejecutar reformas claves para la redistribución y la participación ciudadana. Pese a las críticas y señalamientos de ilegalidad, el éxito conseguido por el fujimorismo en términos de estabilización económica y la cerrada defensa de las élites del modelo neoliberal en un escenario de fragmentación del campo popular, llevó a que tras la caída del régimen, en la denominada "transición", no se incluyera regresar a la Constitución de 1979 ni mucho menos convocara una nueva Asamblea Constituyente conservándose la Carta de 1993 con algunos cambios aprobados por el legislativo. Asimismo, la discusión política sobre el modelo económico quedó fuera de toda negociación y conservó las restricciones constitucionales a cualquier posible intento de modificatoria. Posteriormente, los sucesivos gobiernos de Alejandro Toledo y Alan García, tampoco cuestionaron el marco legislativo heredado del fujimorismo, principalmente en lo referido a la política económica, centrando sus esfuerzos más bien en impulsar programas sociales focalizados para combatir la pobreza. Dada la actual composición del Congreso, la poca voluntad del Partido Nacionalista y la fuerte presencia de lobbies empresariales es poco probable que ocurran cambios normativos relevantes en el corto plazo.

Por otra parte, en lo que respecta a la preeminencia de un discurso autoritario conservador elaborado sobre la historia reciente de violencia y posterior pacificación, el legado fujimorista se presenta dominante en tanto haber posicionado una memoria oficial sobre las causas de la violencia, los artífices de la pacificación y la exaltación del orden y la gobernabilidad como garantía de crecimiento. La afirmación de este discurso desde el Estado tiene claras repercusiones en materia de impunidad y en la forma de enfrentar la protesta social criminalizándola, reprimiéndola y acusando ligeramente de "terroristas" a quienes se oponen o movilizan contra el modelo. Dicho consenso autoritario coincide estrechamente con el sustento ideológico neoliberal articulado por economistas como Hernando de Soto, quien presenta una lectura atractiva del país para las mayorías pues centra las posibilidades de ascenso social en la competencia, las 
opciones del mercado y la exaltación del logro individual. Esto en desmedro de apuestas colectivas y referentes discursivos que valoran la solidaridad y la reciprocidad exponiéndolas interesadamente como prácticas arcaicas y opuestas al desarrollo. La crisis de los partidos de izquierda facilita la hegemonía de este discurso, al ser incapaces de reorganizar estructura política pero sobre todo de articular referentes de sentido que ofrezcan alternativas de vida y den lugar al "mito movilizador" al que aludía José Carlos Mariátegui, hace ya casi un siglo.

La vigencia de estos tres aspectos evidencia entonces una notable ventaja del fujimorismo y los sectores dominantes en cuanto a delinear y consolidar las bases económicas, legislativas e ideológicas sobre las que discurre la política en el Perú de hoy, pues salvo algunas puntuales modificaciones, los posteriores gobiernos las han mantenido y hasta profundizado. A la par, dichos factores presentan importantes ejes de confrontación para las demandas de los movimientos sociales, y se ha exhortado a pensar alternativas que trasciendan la reivindicación inmediatista y avanzar en proponer otras formas de gestionar la economía, los recursos naturales y la reproducción de la vida misma.

\section{LoS Movimientos SOCiales en el Perú DE HOY}

Tras un periodo de repliegue y desmovilización motivado por el cruento conflicto armado que atravesó el país, la política de pacificación fujimorista y el impacto de las reformas estructurales, surgen a fines de los noventa nuevas iniciativas de movilización. Se trata de un proceso complejo de rearticulación, pues han cambiado también las lógicas organizativas, fuertemente influidas por el clientelismo fujimorista y la expansión de Organizaciones no Gubernamentales (ONG) que operan con fondos de la cooperación internacional, ambas con consecuencias directas en las dinámicas políticas de los actores. De este modo, cobran relevancia nuevos espacios cuya acción y organización responde directamente a este pasado reciente y a los cambios ocurridos en el mundo del trabajo, la utilización de los recursos naturales y el manejo macroeconómico derivado del neoliberalismo. Nos referimos a las comunidades y pueblos indígenas que se movilizan contra la expansión minera y petrolera, y manifiestan discursos, iden- 
tidades y demandas contrapuestas al actual modelo, así como otras formas de concebir la relación con la política representativa.

Previamente, conviene señalar que al hablar de movimientos sociales nos referimos a los "procesos por los cuales los actores colectivos producen la acción, siendo capaces de definirse en ella a sí mismos y al campo de acción en que operan". Construyen así su ser social de manera colectiva, lo cual implica constituir una identidad basada en el reconocimiento de intereses compartidos y la definición de estrategias conjuntas a desplegar en la acción colectiva a fin de conseguir los objetivos propuestos. Los movimientos articulan también niveles de organización y liderazgos que les permiten desplegar la protesta, planteando discursos que presentan su problemática e interpelan tanto al Estado como a la sociedad respecto a la necesidad de modificar leyes y políticas públicas e impactar en sentidos comunes e idearios. Actualmente, en Latinoamérica podemos ubicar a los movimientos sociales en el marco de los cambios suscitados a raíz de las "transiciones democráticas" y los procesos de ajuste estructural que significaron las reformas neoliberales. Destaca así por un lado, la primacía del modelo primario exportador pues la presión sobre los recursos naturales da lugar a nuevos "lenguajes de valoración" que colocan al centro la defensa de la territorialidad como espacio de construcción de la comunidad de vida. ${ }^{5}$ Por otro lado, vemos que lejos de esperar que las estructuras partidarias recobren la vitalidad perdida, organizaciones y movimientos sociales tejen diversas interacciones con la política electoral. Es el caso de lo ocurrido en Bolivia donde el movimiento indígena y cocalero impulsó el Movimiento al Socialismo (MAS) como el "instrumento político", lo cual llevó al poder a Evo Morales. Fue también la idea del Partido del Trabajo (PT) en Brasil que articuló una serie de organizaciones con el fin de competir de manera electoral.

Al efectuar un análisis de los principales movimientos sociales en el Perú de hoy, con base en sus plataformas reivindicativas, marcos identitarios y estructuras

4 Alberto Melucci, Acción colectiva, vida cotidiana y democracia, México, El Colegio de México, 2010.

5 Maristella Svampa, Cambio de época movimientos sociales y poder político, Buenos Aires, Siglo xxI/Clacso, 2009. 
organizativas, podemos distinguir dos grandes grupos. Primero los movimientos cuya problemática se enfoca en cuestiones más bien sectoriales y económicas desde un lenguaje de izquierda y estructura sindical (universitarios, docentes, campesinos, médicos, etc.). Por otra parte, las diversas poblaciones que se organizan contra la expansión de la industria extractiva, en especial minera y petrolera, apelan para ello a revaloraciones étnicas y defensa de los recursos con base en una organización más bien territorial (Cajamarca, Espinar, Islay, etc.). Ambos grupos, surgen o se reorganizan en la última década, y expresan un profundo malestar frente a la continuidad de las políticas neoliberales implementadas durante el fujimorismo y la demanda de transformaciones sustanciales a la normatividad y el marco ideológico predominante.

Respecto al primer grupo, tenemos aquí diversas organizaciones sindicales vinculadas a alguna rama del sector público. Es el caso por ejemplo del Sindicato de Trabajadores de la Educación o la Federación Médica, por anotar algunos de los que más se han movilizado en los últimos años reclamando mejoras salariales pero también políticas educativas y de salud que los incluyan como interlocutores. Junto a ellos puede incluirse a los trabajadores de los denominados "services" o empresas de tercerización de servicios, las cuales se expandieron tras la reforma laboral de 1992, lo cual precarizó el empleo y recortó derechos como vacaciones y horas extras, entre otros. Medianas y pequeñas empresas en expansión, como los textiles Topy Top o la fábrica de bebidas Kola Real, se han valido de esta forma de contrato para incrementar sus ganancias, con ello deben enfrentar ahora los reclamos del personal. Finalmente, se ubican los estudiantes universitarios que en diferentes lugares del país protestan ante el abandono en que sobrevive la universidad pública, tras años de recortes presupuestales y represión política durante los años de conflicto armado y posterior pacificación. Si bien a primera vista podía afirmarse que se trata de demandas "economicistas" que se reducen al aumento de sueldos, salarios o presupuestos, la acción de estas organizaciones remite a problemas de fondo relacionadas con la continuidad del modelo económico, al poner en debate el papel del Estado y del mercado en ámbitos como la educación y la salud. Asimismo, plantean impugnaciones concretas tanto al marco normativo heredado del fujimorismo, con una legislación 
laboral abiertamente favorable a los empresarios, como a las inequidades derivadas de una política económica que, pese al crecimiento sostenido del PIB, no resuelve problemas de exclusión ni garantiza derechos para las y los trabajadores.

Tanto los sindicatos mencionados como los estudiantes de las universidades públicas que protestan podrían ubicarse cercanos a una identidad clasista sindical, más politizada y cercana a los partidos que hasta fines de la década de los ochenta conformaron el frente electoral Izquierda Unida. Posteriormente, en el contexto de represión y violencia, tanto los sindicatos como los partidos políticos de izquierda a los que se encontraban vinculados perdieron bases y se entramparon en pugnas internas muchas veces con fuerte sesgo ideológico. Contribuyó a dicha crisis la ausencia de recambio generacional así como el constante desprestigio de los medios de comunicación y sectores empresariales. No obstante, vale mencionar la renovada capacidad de movilización de estos sectores y sus esfuerzos por tender puentes con nuevos grupos de trabajadores como los de servicios, que politizan la lucha por mejoras salariales y laborales y la contextualizan en el marco de la crítica a la continuidad del neoliberalismo en los últimos veinte años.

En el segundo grupo se ubican los pueblos indígenas y comunidades afectadas por la industria extractiva, los mismos que han protagonizado intensas y masivas protestas en puntos tan disímiles del territorio nacional como Cajamarca, Arequipa, Piura o la Amazonía. Se trata de diversas poblaciones que organizan la acción colectiva frente al avance de proyectos mineros o de hidrocarburos, ensayando estructuras organizativas, redes de solidaridad y liderazgos tanto locales como nacionales. Las reivindicaciones de este movimiento se vinculan más bien a la defensa del territorio como espacio de reproducción de la vida, por lo que presentan un fuerte énfasis identitario desde lo étnico y comunitario, y señalan a la vez como principal antagonista al capital transnacional, por la afectación - o amenaza de afectación- socioambiental, la contraposición de cosmovisiones, modelos de vida y formas de propiedad. Aquí se encuentra en primer lugar la Asociación Interétnica de la Selva Peruana (AIDESEP) que agrupa diferentes federaciones locales y regionales de las distintas etnias de la Amazonía con amplia representatividad y capacidad de convocatoria tal como se eviden- 
ció en los paros amazónicos de 2008 y 2009 contra una serie de decretos que facilitaban la inversión extractiva en la selva. En el área andina, aunque la fragmentación de organizaciones es mayor, se puede ubicar al Frente de Defensa de Tambogrande en Piura que consiguió expulsar a la compañía minera canadiense Manhattan, el Frente de Defensa de Islay en Arequipa que se opone al proyecto minero Tía María de la Southern Perú, y el Frente de Defensa de Cajamarca que lidera la lucha contra el proyecto minero Conga de la corporación Newmont. Si bien existe la Confederación Nacional de Comunidades Afectadas por la Minería (CONACAMI), ésta no ha logrado posicionarse como el referente organizativo nacional.

En lo referido a la identidad colectiva articulada por estos grupos, conviene destacar la crítica al modelo de desarrollo predominante que plantean, al denunciar la desmedida extracción de los recursos naturales ubicados en los territorios que ocupan y el avance de la lógica del mercado para comprar, vender y concesionar la tierra y también sus conocimientos. Se expone así un profundo cuestionamiento a la acción del Estado que, lejos de ser un actor imparcial, favorece, promueve y defiende esta dinámica de desarrollo al argumentar lo mucho que estas empresas benefician al país y mostrar cifras de crecimiento macroeconómico. No obstante, los pueblos y comunidades de la sierra y la Amazonía no han mejorado de manera sustancial sus condiciones de vida, pues persisten altas tasas de pobreza y extrema pobreza. Tal situación de exclusión es problematizada por estos movimientos sociales, que configuran un horizonte crítico de gran potencialidad transformadora pues además de exigir mejoras redistributivas, cuestionan esta forma de "progreso" asociada al neoliberalismo. Si bien persiste todavía una fuerte fragmentación con directivas poco consolidadas y débiles articulaciones nacionales, resalta la capacidad de estos actores para desafiar el consenso autoritario posfujimorista, articulando diversas estrategias organizativas y repertorios de protesta que han logrado detener importantes proyectos extractivos e iniciativas legislativas.

Ambos grupos de movimientos han incorporado a sus estrategias de acción la participación en el terreno político institucional a fin de lograr puestos de representación que les permitan concretar sus plataformas desde el ámbito de la 
gestión. No obstante, vamos a priorizar el análisis de los movimientos vinculados a los denominados conflictos socioambientales tanto por su potencial para interpelar las dimensiones económicas, normativas e ideológicas dominantes como por los esfuerzos de desarrollar otras formas de organización y representación política más ligadas a lo asambleario territorial. Los gremios y sindicatos, han mantenido el vínculo con partidos de izquierda y en periodos electorales suelen presentar a dirigentes suyos como candidatos en estos grupos, mientras que los pueblos y comunidades afectados por la expansión extractiva ensayan otras alianzas y acercamientos con los actores políticos. El énfasis en la participación y la consulta, tanto como la desconfianza frente a los actores políticos, son algunas características que marcan esta etapa, la cual da lugar a encuentros y distanciamientos que marcan la pauta de nuevos desafíos.

\section{DiNÁMICAS POLÍTICAS, EPISODIO UNO:}

\section{ACERCAMIENTOS Y REPRESENTACIONES}

Tal como se mencionó, las acciones de los movimientos sociales, en especial pueblos indígenas y comunidades afectadas por la industria extractiva, han incluido también la opción de competir electoralmente y lograr puestos de representación política en las escalas local, regional y nacional. Son varios los puntos de evaluación tomados en cuenta para tal decisión. Al parecer, existe la percepción compartida de que la movilización da resultados, y así lo demuestra la paralización de proyectos mineros importantes como Tambogrande o Tía María. No obstante, esto ocurre a costa de un gran despliegue de recursos humanos y materiales, así como de articulaciones locales, nacionales e internacionales. Por otra parte, los resultados obtenidos generalmente no son definitivos pues tienen que ver con políticas nacionales y aunque se logre detener el proyecto no se anula la concesión pues queda latente la posibilidad de que otra empresa pueda retomar la iniciativa a la postre. Finalmente, la dinámica misma de construcción interna del movimiento y su arraigo territorial lleva a plantearse con mayor sistematicidad desarrollar experiencias de gobierno local, y participar en elecciones de municipios 
distritales y provinciales ante la posibilidad de ensayar desde ahí proyectos productivos, de educación o salud de corte más intercultural.

Para concretar la participación política electoral, las vías contempladas por los movimientos han sido diversas y los resultados no siempre favorables, pero han significado importantes procesos de aprendizaje, interacción y acercamiento con actores institucionales, sobre todo partidos políticos y organismos electorales que regulan la participación. A partir del 2006, se identifican con mayor claridad procesos de debate interno que devienen en la decisión de participar en el terreno electoral. No es que antes no haya ocurrido, lo más probable es que sí, pero ahora la decisión parte del grupo y no necesariamente responde a la invitación del partido como sucedía antes. Es la organización la que decide cómo, con quién y para qué participar en política.

Un caso importante de esa etapa es el movimiento cocalero, el mismo que decide participar electoralmente pero no logra acuerdo respecto a la mejor fórmula y se divide en dos grupos distintos. Por un lado, los cocaleros del Valle del río Apurímac, que optan por construir el "instrumento político", formando el partido Qatun Tarpuy, el cual logró la elección de dirigentes cocaleros directamente vinculados al movimiento como regidores y alcaldes distritales. Por el otro, los cocaleros del Huallaga que concretan una alianza con el Partido Nacionalista y aprovechan su inscripción para colocar candidatos distritales al Parlamento Nacional. ${ }^{6}$ Otro caso relevante es el del movimiento indígena amazónico, que desde sus distintas bases regionales vinculadas a AIDESEP, deciden participar en la coyuntura electoral, principalmente en municipalidades distritales y provinciales de mayoritaria población indígena. Así lo hace por ejemplo el pueblo ashaninka en los distritos de Pangoa y río Tambo en la selva central donde líderes vinculados al movimiento son elegidos alcaldes, o el de los distritos de Nieva y el Cenepa en Amazonas, donde ocurre lo mismo con los dirigentes awajum y wampis.

6 "Movimiento cocalero, política y representación: los casos boliviano y peruano", en Mayarí Castillo y Anahí Durand, Identidades, etnicidad y racismo en América Latina, Quito, Flacso, noviembre de 2008. 
Sería extenso analizar aquí cómo se ha desarrollado la actuación de los movimientos sociales en la gestión del poder en las escalas municipal, regional o parlamentaria y amerita un estudio de mayor profundidad. En líneas generales destaca el esfuerzo desplegado por los líderes electos y sus organizaciones tanto para lograr ganar las elecciones como para una vez en ejercicio, desarrollar gestiones más vinculadas a las dinámicas y necesidades de la población en los territorios donde son elegidos. Sin embargo, tanto en el caso de los alcaldes cocaleros como en el de las autoridades indígenas amazónicas, quedó en evidencia también el poco margen de acción para establecer políticas y proyectos de envergadura y el escaso margen para incidir en la política extractiva que los afecta directamente. Asimismo, las autoridades vinculadas al movimiento enfrentan una doble presión, tanto por parte de las bases y pobladores que les exigen mantenerse al frente de sus demandas incluso como medidas de fuerza, como por parte del poder ejecutivo que les reclama anteponer su condición de representantes del Estado. La intención del gobierno de frenar la actuación de estas autoridades en el terreno contencioso se ha valido además de la criminalización, llegando a penalizar la participación de autoridades electas en movilizaciones y protestas.?

Durante el año 2011 la coyuntura electoral nacional se mostraba como oportuna y relevante para que los movimientos sociales ensayaran estrategias y mecanismos en pos de ocupar puestos de representación en el Congreso. Dado el panorama político de entonces, Gana Perú, partido liderado por Ollanta Humala, se presentaba como el líder de la oposición enarbolando un discurso abiertamente crítico al modelo neoliberal y a la continuidad que representaban los candidatos de la derecha y centro derecha encarnada por sus principales competidores, el ex presidente Alejandro Toledo y la hija del ex presidente Fujimori, Keiko Sofía Fujimori. El cuestionamiento a la clase política realizado por Humala y su énfasis en la necesidad de realizar reformas a la política económica y extractiva encontró gran empatía con la postura de los movimientos sociales. Dicha

El 22 de julio de 2007 se promulgó el D.L núm. 982 que modifica el Código Penal y plantea - entre otros puntos - que las autoridades o funcionarios públicos que participen en huelgas o protestas serán sancionados con inhabilitación y penas de cárcel. 
coincidencia de plataformas y la ausencia de otras opciones transformadoras con opciones de triunfo, resultaba ventajosa para congregar el voto opositor pero hacía menos necesario suscribir alianzas y compromisos formales con las principales organizaciones sociales movilizadas. De este modo, no se concretaron acuerdos con AIDESEP que pretendían la candidatura parlamentaria de su presidente Alberto Pizango, ni con la CGTP que esperaba postular a su secretario general Mario Huamán. También quedaron fuera de la lista parlamentaria líderes locales representativos de la lucha contra la minería en Arequipa o Cajamarca. Al intuir que de todas formas las bases de estos movimientos acabarían votando por Gana Perú, el humalismo prefirió no generar compromisos formales con sectores que podían resaltar la imagen de "radical" que presentaba la prensa y lo alejaba de las preferencias electorales de las clases medias urbanas necesarias para llegar al gobierno.

Los sectores sociales movilizados, específicamente las comunidades afectadas por la industria extractiva y el movimiento indígena-amazónico, no lograron articular una plataforma conjunta que ampliara sus posibilidades de exigir un trato con Gana Perú en mayor igualdad de condiciones. Mientras AIDESEP concentraba esfuerzos en inscribir su propio partido y los diversos frentes de Defensa tanteaban acercamientos a grupos políticos minoritarios, se dejó de lado la posibilidad de consolidar una propuesta unitaria. Ya ad portas de las elecciones, las organizaciones ensayaron acercamientos con Humala por separado, cada cual con sus líderes y agendas, y obtuvieron del candidato apenas algunos compromisos generales. Los resultados electorales acabaron por demostrar que, en efecto, Ollanta aglutinó el voto de los sectores movilizados, en un contexto de disputa altamente polarizado. Una forma de aproximarnos a este encuentro de los movimientos y de Gana Perú es analizando los votos obtenidos por los partidos políticos en las provincias y distritos donde esos movimientos tienen mayor presencia. En este sentido, nos centramos en las localidades donde se desarrollaron protestas emblemáticas y también donde se ubican conflictos en estado latente.

En el caso del movimiento indígena amazónico, tomamos en cuenta tres criterios para seleccionar los ámbitos de votación; el primero, las provincias más movilizadas durante los paros amazónicos de 2008 y de 2009; el segundo, aque- 
llas que presentan mayor cantidad de población nativa, y el tercero los lugares donde existen conflictos latentes con actividades extractivas que incluyen hidroeléctricas y forestales. De modo general podemos afirmar que en la mayoría de provincias el triunfo de Gana Perú es claro. En las provincias de Bagua y Condorcanqui en la región de Amazonas los resultados fueron contundentes al influir la incorporación en la lista parlamentaria de Eduardo Kayap, pastor evangélico awajum no directamente vinculado a AIDESEP pero igual es elegido congresista por Amazonas. En ambas provincias la Fuerza 2011, liderada por Keiko Fujimori, queda en segundo lugar pero a una distancia larga, y más lejos aún los otros candidatos. Es distinto el caso de la selva central (Satipo, Oxapampa, Chanchamayo): el único espacio donde el fujimorismo obtiene el primer lugar con porcentajes significativos. Tal aceptación fujimorista en una zona fuertemente afectada por el conflicto interno armado, debe guardar relación con la memoria de Alberto Fujimori como el artífice de la pacificación que apoyó a los Comités de Autodefensa y posteriormente mantuvo su presencia con base en medidas clientelares.

Gráfica 1. Resultados electorales presidenciales en provincias amazónicas, 2011.

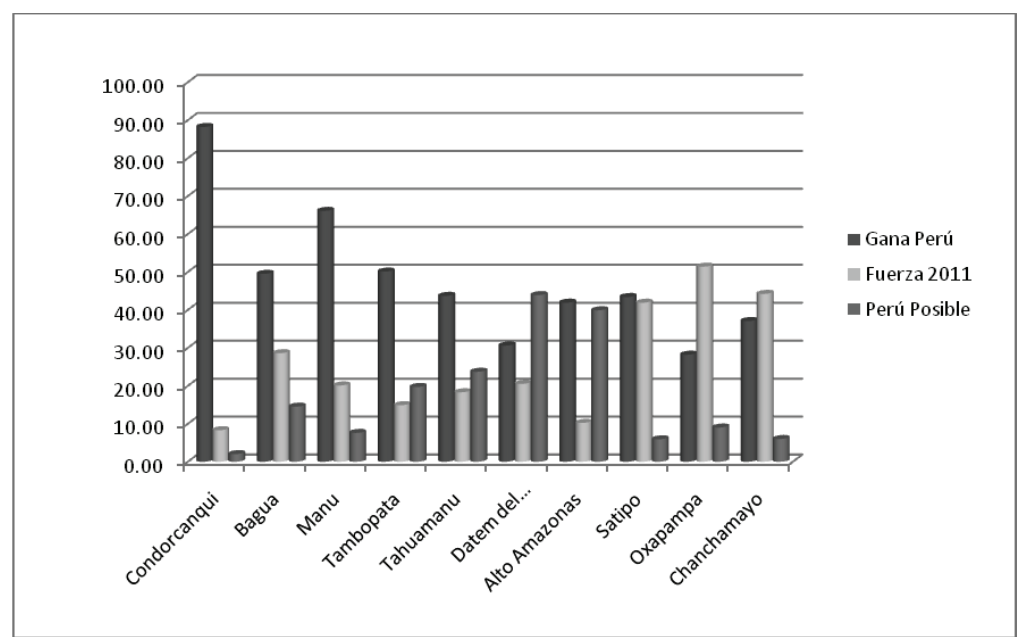

Fuente: Elaboración propia con base en www.onpe.gob.pe/. 
En el caso de las comunidades afectadas por la minería ubicadas en los Andes, tomamos como referencia algunas provincias donde se encuentran proyectos extractivos relevantes que han suscitado protestas o donde hay una situación de conflictividad latente. Nos referimos a los proyectos mineros de Tía María en Islay (Arequipa), Yanacocha en Hualgayoc, incluyendo el proyecto Conga (Cajamarca), Pampamali en Angares (Huancavelica) y Río Blanco en Huancabamba (Piura). Hemos incluido también la provincia de Espinar, donde en el 2012 sucedieron violentos eventos de protesta contra la minera Billington Tintaya, pues exigían una mejor distribución de las ganancias y mayor control ambiental. En estas provincias hubo coincidencias con las tendencias nacionales, Gana Perú es el rotundo ganador de la contienda con porcentajes que en casi todos los casos supera la mitad del electorado.

Gráfica 2. Resultados electorales presidenciales en las provincias andinas, 2011.

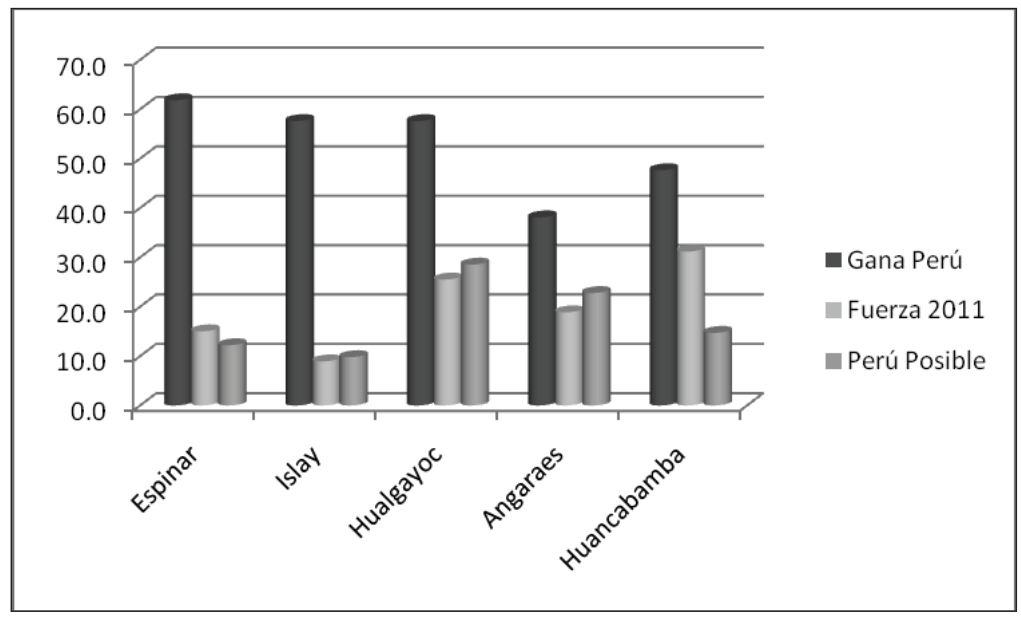

Fuente: Elaboración propia con base en www.onpe.gob.pe/. 
Finalmente, en todas las provincias de mayor producción de hoja de coca, Ollanta Humala tambien es el ganador, al obtener los porcentajes más altos en las provincias del Valle del Río Apurímac Ene (VRAE) como son Huanta, La Mar y La Convención, donde supera 50\% de los votos. En las provincias ubicadas en el valle del Huallaga (Huamalies, Leoncio Prado, Tocache, Mariscal Cáceres y Coronel Portillo) también resulta ganador el partido de Humala, pero la distancia frente a sus competidores es menor, al reducirse la ventaja frente al fujimorismo o al partido Perú Posible del ex presidente Toledo.

Gráfica 3. Resultados electorales presidenciales en las provincias cocaleras, 2011.

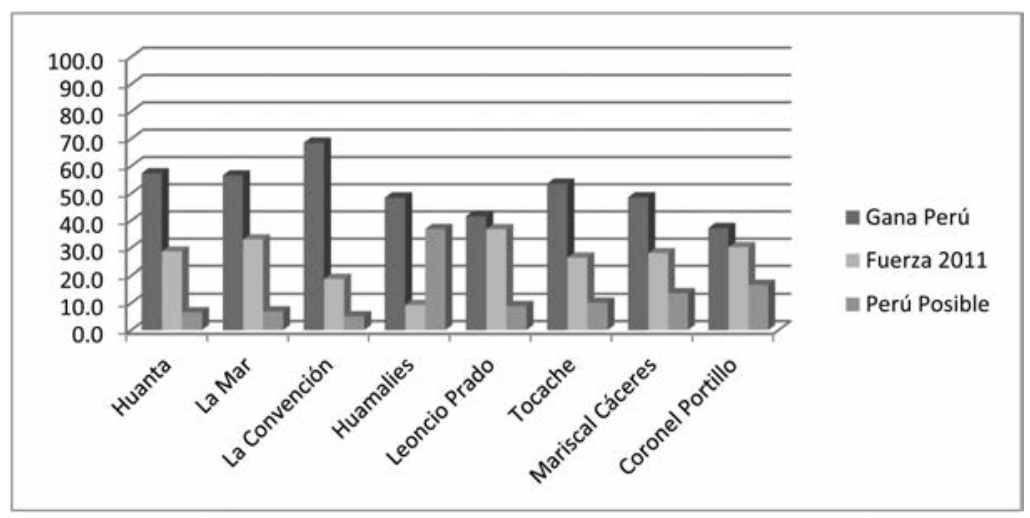

Fuente: Elaboración propia con base en www.onpe.gob.pe/.

En general, los resultados electorales de 2011 demuestran la identificación y cercanía lograda entre los movimientos sociales y Ollanta Humala, por lo menos en términos de adhesión electoral, la misma que se mantuvo en el tiempo pues en 2006 también estos sectores le otorgaron su voto. Así, la elección de Gana Perú y la coalición de centro izquierda que lo acompañó los primeros seis meses de gobierno liderada por el ex premier Salomon Lerner, fue el punto culminante de un proceso de acercamiento y coincidencia por posicionar en el escenario nacional posturas críticas al modelo neolibral. Resume también las 
expectativas y esfuerzos de los movimientos por lograr representación política, no tanto eligiendo autoridades directamente vinculadas al movimiento, cosa que no consiguen autonómamente ni Humala les facilita, pero sí en términos de identificación con el nuevo presidente y la confianza en que "representa sus intereses". No obstante, a los pocos meses de que Humala asumiera el cargo, el estallido de nuevos conflictos socioambientales puso en evidencia la opción del nuevo gobierno por continuar el modelo neoliberal, con ello se inició una nueva etapa de relación de los movimientos con la política institucional.

\section{DiNÁMICAS POLÍTICAS, EPISODIO DOS:}

\section{VUELTA AL DISTANCIAMIENTO Y OTROS CAMINOS}

De manera temprana, en noviembre de 2011, las protestas en Cajamarca contra el proyecto minero Conga, a cargo de las empresas Newmont y Yanacocha, originaron una crisis política que culminó con la salida del presidente Lerner, la ruptura de Humala con sus aliados de izquierda y el realineamiento con la continuidad de la política posfujimorista al asumir sus dimensiones políticas, económicas y sociales. Con ello, se definió a la vez una nueva etapa de distanciamiento entre los movimientos sociales aquí mencionados y los actores políticos institucionales, en especial el Partido Nacionalista o su versión electoral Gana Perú. Una vez dejadas de lado las promesas de introducir reformas sustanciales al modelo neoliberal -y en particular a su componente extractivo- los pueblos y comunidades movilizados, así como las organizaciones sociales, vieron clausurada la posibilidad de contar con un gobierno que representara sus demandas. Además, este cambio de plataforma por parte de Humala fue percibido como una muestra del poder ejercido por los grupos empresariales nacionales y transnacionales, que finalmente no necesitan de un candidato "propio" para hacer prevalecer su agenda e intereses.

La postura asumida por el gobierno generó una intensa reacción en distintas localidades donde existen importantes proyectos o concesiones mineras. Las protestas en las provincias de Hualgayoc y Celendin en Cajamarca contra el proyecto Conga, que pretende desecar tres lagunas naturales para extraer oro 
a tajo abierto, y las protestas en la provincia de Espinar en el Cusco contra la acción contaminante y la escasa redistribución por parte de la minera Billington Tintaya pueden considerarse las más representativas de esta nueva etapa. En ambos casos, las protestas se enmarcaron además en amplios diagnósticos preexistentes que señalaban los pasivos y problemas más urgentes que el nuevo gobierno debía abordar para paliar el conflicto socioambiental. ${ }^{8}$ Según estos informes, un primer grupo de reformas pendientes se refería al manejo ambiental de las concesiones mineras, principalmente las pautas de aprobación de los Estudios de Impacto Ambiental (EIA) que originaban diversas denuncias a las empresas por manipular las audiencias y a los funcionarios por su abierta parcialidad con los inversionistas. En un segundo grupo se contaban los cambios relacionados a la distribución de ganancias y beneficios de las empresas mineras y petroleras. En los niveles local y provincial, las empresas habían firmado diversos convenios sobre entrega de fondos que la población pedía renegociar. Un reclamo legítimo teniendo en cuenta que si los precios del mineral y las ganancias de la empresa subían, los montos pactados también podían revisarse. Además se exigía mayor autonomía para el uso de estos beneficios, pues las empresas suelen tutelar su dinero canalizándolo a través de fundaciones y ong. Después de un año de gestión humalista, según los reportes de la Defensoría del Pueblo, los conflictos socioambientales continúan dominando la escena y no han disminuido significativamente respecto al gobierno anterior. De este modo, en julio de 2011, Defensoría del Pueblo reportó 118 conflictos socioambientales activos (55\%) del total de casos, para julio de 2012 aumentó a 148 conflictos activos, poco más de $60 \%$ del total.

8 En www.defensoria.gob.pe, Verónika Mendoza,"La génesis de los conflictos", en Revistaideele.com, Lima, 29 de noviembre de 2011. En http://www.revistaideele.com/content/la-g\%C3\%A9nesisde-los-conflictos. 
Gráfica 4. Cuadro de conflictividad, julio de 2012.

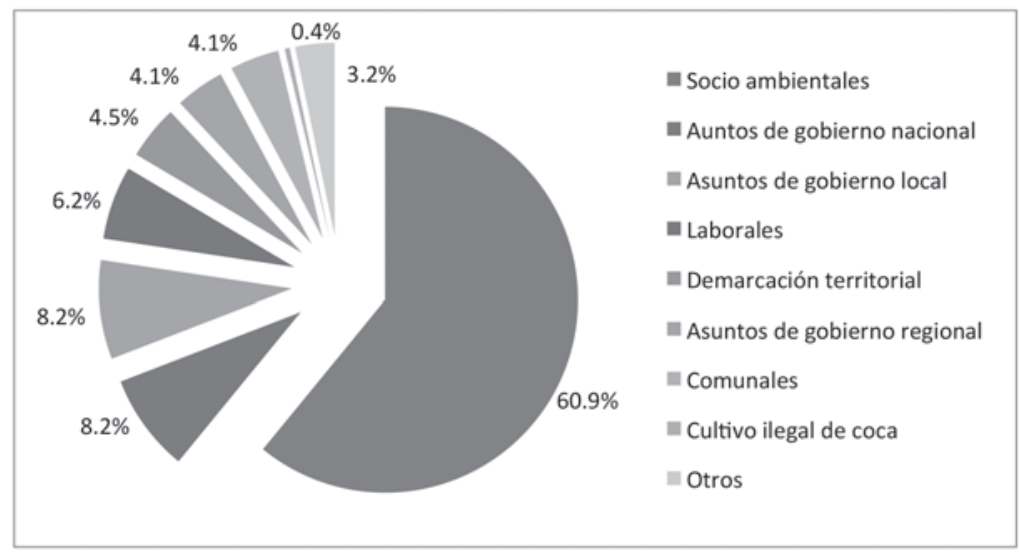

Fuente: www.defensoria.gob.pe.

¿Qué es lo que revelan estas nuevas movilizaciones en términos de la interacción de las organizaciones sociales con el Estado y los actores institucionales? Durante las protestas de Cajamarca y Cusco quedó claro que el Estado estaba dispuesto a llevar la defensa de la política extractiva hasta sus últimas consecuencias, asumiendo la misma represión y criminalización ya ensayada por el ex presidente García. Esto implicaba desde acusaciones a dirigentes y autoridades de terroristas o politiqueros, hasta constantes alusiones a la población como "gente manipulada y desinformada". Además, el nuevo primer ministro, Óscar Valdés, asumió la estrategia de presionar a los gobiernos subnacionales que apoyaban los reclamos de la población, al ordenar al Ministerio de Economía y Finanzas "bloquear" las cuentas del Gobierno Regional de Cajamarca y de la municipalidad provincial de Espinar, y encarcelar además al alcalde de Espinar, Óscar Mollohuaca, y los principales líderes acusados de alterar el orden público. Las sendas declaraciones del estado de sitio en Espinar (Cusco), Celendin y Bambamarca (Cajamarca) y la represión policial, dejaron un saldo de cientos de heridos, dos muertos en Espinar en mayo de 2012 y cinco fallecidos en Cajamarca 
en el mes de julio de 2012. ${ }^{9}$ La consecuencia de esta tensa coyuntura fue la división de la bancada nacionalista en el Congreso que terminó por alejarse del equipo de izquierda que acompañó a Humala, elaboró el Plan de Gobierno y encabezó su primer gabinete.

Dichas protestas demostraron la improvisación del gobierno, sin bases regionales propias ni cuadros dirigenciales eminentes capaces de reemplazar a quienes se retiraron básicamente por las discrepancias en el manejo de la política económica y socioambiental. Revelaron también a un presidente dispuesto a ceder rápidamente a las presiones de la Sociedad Nacional de Minería, a un hombre bastante pragmático y mucho más empeñado en fortalecer su proyecto partidario familiar, junto a la primera dama, quien estaba decidido a construir una nueva correlación progresista. El gobierno de Humala tampoco ha sido capaz de brindar un horizonte claro del proyecto que quiere para el país capaz de disputar los pilares ideológicos y de sentido del posfujimorismo; más allá del eslogan de la "inclusión" no hay un mensaje político sustancial respecto al desa rrollo, la modernización o los derechos de los pueblos y comunidades indígenas que el modelo no ha podido sacar de la pobreza. Presentar la inclusión vinculada a los programas sociales, aunque es importante resulta insuficiente, pues apela a la focalización en desmedro de derechos universales y de reconocimiento político priorizando casi exclusivamente la lucha contra la pobreza sin combatir antiguas y reiteradas inequidades. A un año de llegar al poder, sin estructura ni operadores partidarios, sin aliados importantes ni cuadros técnicos, sin referentes ideológicos que contraponer al neoliberalismo, el gobierno del presidente Humala luce desgastado y como "más de lo mismo" tanto para la derecha que temía su llegada al poder como para las organizaciones y los sectores populares que lo respaldaron.

Desde las organizaciones sociales y poblaciones movilizadas, estas protestas marcan el inicio de un nuevo periodo de distanciamiento frente a los actores político-institucionales, en particular el Partido Nacionalista que logró aglutinar

9 Véase al respecto Diario La República, Lima, julio de 2012. En http://www.larepublica.pe/0507-2012/gregorio-santos-ya-son-5-los-muertos-por-protestas-en-cajamarca. 
electoralmente sus críticas y expectativas de cambio frente al modelo. Revelan además la decisión de asumir otros caminos y nuevos desafíos, con miras a hacer valer sus demandas y propuestas políticas. Destacaría así la reafirmación por la vía contenciosa, donde las bases son las que deciden continuar las protestas sobrepasando con frecuencia la representatividad de los líderes, los cuales deben convocar permanentemente a consulta y reconocer a la Asamblea como única instancia de decisión. Esta reafirmación, se encuentra avalada por la comprobación real de que, dados los candados legales y hasta constitucionales existentes, sólo con la protesta se consigue poner límites al capital extractivo; finalmente el proyecto Conga está detenido y la Mesa de Negociación de Espinar consiguió nuevas evaluaciones sobre los efectos contaminantes de la empresa minera.

Cabe resaltar también que las últimas protestas expresan un persistente malestar con la política económica y el modelo de desarrollo primario exportador, pues pese a todas las promesas de crecimiento no ha logrado articular su dinámica a los pueblos y comunidades donde se ubican los recursos. Esto no sólo por problemas de redistribución económica sino por la existencia de formas de vida y cosmovisiones respecto a la naturaleza y la vida comunal no reducibles a la lógica costo-beneficio del mercado; en Cajamarca la empresa Yanacocha en reemplazo de las lagunas naturales ha ofrecido construir tres reservorios que, según ellos, triplicarán el abastecimiento de agua en la zona. La prensa limeña y el mismo Estado descalifican a los campesinos por no ceder ante dichos ofrecimientos, desprecian la valoración que tienen por el ecosistema y reproducción de la vida cotidiana que se origina en torno a este. Finalmente, no se puede dejar de mencionar que las protestas también pusieron en evidencia la fragmentación de las luchas socioambientales en el país, expresada en plataformas poco cohesionadas, liderazgos locales tendientes al caudillismo y grandes dificultades para dotar a las protestas de carácter nacional de modo que, por ejemplo, lo actuado en Cajamarca pudiera tener alguna repercusión en Cusco. Esta dispersión puede responder tanto a fuertes anclajes territoriales y dinámicas organizativas diferenciadas, cuanto a la presencia de sectores interesados en conseguir réditos políticos, levantando para ello posturas maximalistas que les pueden brindar adhesiones y mayor protagonismo faccionalista o 
personal. Esta etapa resulta un desafío para los movimientos, cuyo desplazamiento busca usufructuar el potencial desplegado, al afirmar más bien las articulaciones horizontales entre las mismas organizaciones opuestas al avance del capital extractivo y otras que, como los mencionados gremios y sindicatos, pueden ampliar la resonancia de sus demandas en un marco de crítica al modelo vigente hace dos décadas.

\section{CONCLUSIONES}

Tal como se ha intentado explicar, el actual momento político interpela a las organizaciones sociales respecto a cómo lograr cambios sustanciales tanto en el modelo de desarrollo como en el proyecto ideológico y en los referentes de sentido posfujimoristas que lo respaldan y que bien pueden leerse como remozados discursos conservadores. Esto supone repensar los acercamientos e interacciones con la política electoral y partidaria y las formas en que se ha desarrollado la articulación programática, organizativa y discursiva al interior del mismo sector de movimientos sociales y del campo popular, incluidos partidos de izquierda. La elección de Ollanta demostró que el apoyo a determinado partido como atajo para conseguir puestos de poder o impulsar reformas no garantizan el respeto de los compromisos electorales, menos aun si el candidato no tiene mayor vínculo o trayectoria de lucha cercana a los movimientos.

No obstante, más allá de las limitaciones y aprendizajes, encontramos dos aspectos importantes de la acción de los movimientos sociales aquí anotados en tanto su proyección para avanzar en desmontar el andamiaje posfujimorista y construir una sociedad más democrática. En primer término destacan sus posibilidades de "representación" que logra poner en escena el descontento frente al modelo. Desde un enfoque posestructuralista, además del hecho de elegir entre partidos y delegar autoridad a los gobernantes, la función de representar abarca la tarea de incorporar demandas marginales que exceden las exigencias de un grupo.$^{10}$ Por ello, una tarea importante del representante es transmitir

\footnotetext{
${ }^{10}$ Ernesto Laclau, La razón populista, México, FCE, 2006.
} 
puntos de identificación a los distintos actores sociales presentes en la sociedad, acortando la distancia entre el interés del grupo y el de la comunidad nacional. Se desarrolla, así, un proceso de homogeneización sustentado en coincidencias e identificaciones, en el que los partidos desempeñan un papel importante pero no son los únicos actores, pues las voluntades colectivas pueden articularse en torno de un líder carismático, una demanda compartida o una organización sectorial. Por ejemplo, durante los años ochenta, en América Latina, el movimiento de derechos humanos logró representar la lucha contra las dictaduras, lo que llevó a la representación política a adquirir un componente de expresividad en el cual el todo se reconocía en alguna de las partes, no necesariamente un partido político. Lo mismo podríamos afirmar del Paro Amazónico de mayo de 2009 y los sucesos de Bagua o de las protestas contra el proyecto Conga en 2011 que consiguieron representar políticamente el descontento de un vasto sector de la ciudadanía con el modelo neoliberal, la depredación de los recursos naturales y la parcialidad estatal frente al capital transnacional extractivo.

Actualmente, los movimientos sociales están desarrollando ya una importante función de "representación expresiva", y transmiten puntos de identificación en una sociedad fragmentada que impugna al Estado y a la sociedad sobre la exclusión y las desigualdades existentes. Justamente, el segundo aspecto a resaltar es el potencial de estos movimientos para protagonizar procesos democratizadores, en el sentido anotado por Di Marco, como esfuerzos por replantear la distribución desigual de los recursos simbólicos y materiales presentes en la sociedad. ${ }^{11}$ Se trata de procesos abiertos que tienen lugar principalmente en los espacios colectivos pero que repercuten en la vida cotidiana, generando experiencias y relaciones por las cuales los sujetos subordinados amplían el reconocimiento de sus derechos y asumen luchar por éstos, cuestionando las relaciones de poder existentes y la legitimidad de ese poder. Los movimientos sociales,

${ }^{11}$ Graciela Di Marco, "Movimientos sociales y democracia radical; lo público y lo privado", en Rafael Hoetmer [ed.], Repensar la política desde América Latina, Lima, Programa Democracia y Transformación Global, 2010. 
particularmente los movilizados contra el capital extractivo, con su accionar y plataformas, cuestionan las desigualdades sostenidas y profundizadas por el modelo, en tanto concentración de las ganancias en unos pocos inversionistas y grupos de poder, depredación de los recursos, afectación a sus territorios, y persistencia de jerarquías étnicas, de clase y de género. Con sus reclamos, trascienden lo meramente economicista, y ejercen formas de decisión, organización y articulación que intentan ser menos verticales y más consensuadas, esforzándose por construir las bases de una sociedad igualitaria, con los mismos derechos y oportunidades para todos.

Tanto la capacidad de representar la disconformidad con el modelo como el potencial democratizador que expresan, hacen de los movimientos analizados actores claves para comprender el curso de la dinámica política en el actual Perú. Queda por vislumbrar cómo las organizaciones, los pueblos y comunidades movilizados asumen este potencial y lo transmiten a la sociedad, articulando nuevas lecturas del país y marcos de sentido capaces de tener eco en el conjunto de la sociedad incluyendo aquella que sí se beneficia del crecimiento. Esto implica "desmontar" la ilusión desarrollista, al evidenciar las limitaciones de un modelo que se sustenta en la exclusión de importantes sectores, que pone en riesgo el futuro de las nuevas generaciones y que ha sido aplicado en el marco del proceso autoritario fujimorista. Requiere además avanzar en formular otras visiones del desarrollo que incluyan la reformulación de la matriz productiva para el país. De qué forma los movimientos logren concretar estas tareas y qué tanto se mantengan en la apuesta por la estrategia dual de la protesta y la competencia por puestos de representación política, son algunos de los procesos que muy probablemente dominarán la nueva temporada política, la cual requerirá de nuevos y más profundos análisis.

Recibido: 26 de febrero, 2013.

Aceptado: 28 de octubre, 2013. 


\section{FUENTES}

Bebbington, Anthony, "Elementos para una ecología política para los movimientos sociales y el desarrollo territorial en zonas mineras", en Anthony Bebbington [ed], Minería, movimientos sociales y respuestas campesinas. Una ecología política de transformaciones territoriales, Lima, Instituto de Estudios Peruanos, 2007.

Castillo Mayarí y Anahí Durand, "Movimiento cocalero, política y representación: los casos boliviano y peruano", en Identidades, etnicidad y racismo en América Latina, Quito, Flacso, 2008 (Col. 50 años).

Di Marco, Graciela, "Movimientos sociales y democracia radical; lo público y lo privado", en Rafael Hoetmer [ed.], Repensar la política desde América Latina, Lima, Programa Democracia y Transformación Global, 2010.

Diario El Comercio.

Diario La República.

Durand, Francisco, "El debate sobre la captura del Estado Peruano", en Eduardo Toche [comp.], Perú boy; la gran continuidad, Lima, Centro de Estudios y Promoción del Desarrollo, 2012.

LACLAu, ERNeSTO, La razón populista, México, FCE, 2006.

Mendoza VeróniKa, "La génesis de los conflictos", en Revista Ideele.com, Lima, 29 de noviembre, 2011. En http://www.revistaideele.com/content/la-g\%C3 \%A9nesis-de-los-conflictos.

Remy, MARÍA ISABEL, "Democracia y desigualdad: poderes fácticos, régimen político y ciudadanías diferenciadas", en Julio Cotler y Ricardo Cuenca [eds.], Las desigualdades en el Perú, balances críticos, Lima, Instituto de Estudios Peruanos, 2011.

Svampa, Maristella, Cambio de época. Movimientos sociales y poder político, Buenos Aires, Siglo xxi/Clacso, 2009. 